\section{Atrophie villositaire intestinale dans un nouveau modèle murin de la maladie coeliaque}

Valérie Abadie
Département de médecine, Service de gastro-entérologie, hépatologie et nutrition, Université de Chicago, KCBD $900 \varepsilon$. $57^{\text {th }}$ street MB9 60637 Chicago, États-Unis. vabadie@medicine.bsd.uchicago.edu
Un modèle murin de la maladie cœliaque : la quête du Graal

La maladie cœliaque est une maladie inflammatoire de l'intestin qui survient en moyenne chez $1 \%$ de la population [1]. Elle se développe uniquement chez des individus génétiquement prédisposés, porteurs des molécules HLA (human leukocyte antigen)-DQ2 ou HLA-DQ8, et qui consomment des protéines de gluten comprenant des prolamines (gliadines du blé et protéines apparentées du seigle, de l'orge et de l'avoine) et des gluténines [1]. Elle se caractérise sur le plan sérologique par la présence d'anticorps dirigés contre les peptides de gluten et contre une enzyme tissulaire, la transglutaminase de type 2 (TG2), et sur le plan histologique par une atrophie villositaire de la paroi duodénale accompagnée d'une hyperplasie des cryptes intestinales et d'une augmentation du nombre des lymphocytes intra-épithéliaux [2]. Le seul traitement disponible à ce jour consiste à suivre un régime sans gluten strict, à vie [3], qui, dans la plupart des cas, conduit à la disparition des symptômes et à une normalisation de l'épithélium intestinal. Or, certains patients conservent des dommages intestinaux malgré ce régime, et peuvent souffrir de complications rares, telles que la sprue réfractaire, qui peut progresser vers un lymphome intestinal [4]. Compte tenu de la difficulté d'adhérer à un régime alimentaire aussi contraignant, et de l'absence d'efficacité de cette diète chez certains patients, la recherche d'un traitement de cette maladie mobilise plusieurs équipes de chercheurs. Cependant, la mise au point de nouvelles stratégies thérapeutiques a été freinée jusqu'à présent par notre compréhension incomplète des interactions cellulaires et des mécanismes menant à l'atrophie villositaire, et par l'absence d'un modèle animal de la maladie.

L'identification de lymphocytes T spécifiques du gluten, restreints par les molécules HLA-DQ2 ou HLA-DQ8 et produisant de l'interféron- $\gamma($ IFN- $\gamma)$, dans le chorion de la muqueuse intestinale chez les patients atteints de maladie cœliaque [5] suggère qu'une réponse pro-inflammatoire spécifique du gluten de type $T_{H} 1$ ( $T$ helper 1 ) joue un rôle clé dans la pathogénèse. L'enzyme TG2, qui est aussi la cible des auto-anticorps, convertit certains résidus glutamine présents dans les peptides de gliadine en glutamate par désamidation, ce qui accroît l'affinité des peptides pour HLA-DQ2 et HLADQ8, et ainsi contribue probablement à l'amplification de la réponse des lymphocytes T CD4 $4^{+}$contre le gluten [6-8]. Cependant, plusieurs éléments indiquent que l'immunité reposant sur les lymphocytes $\mathrm{T} \mathrm{CD4}^{+}$spécifiques du gluten n'est pas suffisante pour promouvoir la destruction tissulaire dans le contexte de la maladie cœliaque. En effet, des souris HLA-DQ8 ou HLA-DR3-DQ2 transgéniques immunisées avec des peptides de gluten en présence d'adjuvant développent une réponse inflammatoire adaptative mobilisant des lymphocytes T CD4 ${ }^{+}$contre le gluten accompagnée de la production d'anticorps, mais pas d'atrophie villositaire [9-12]. Malgré la prise en compte de l'absolue nécessité d'être porteur du «bon » haplotype HLA et d'être exposé au gluten pour développer la maladie cœliaque, les résultats obtenus dans ces premiers modèles murins suggèrent qu'un facteur additionnel est requis pour que la maladie évolue d'une forme silencieuse vers une forme active avec destruction du tissu intestinal.

Ce facteur pourrait être le stress épithélial, accompagné d'une augmentation de l'expression de la cytokine pro-inflammatoire interleukine (IL)-15. Ainsi, il a été montré que le transfert de lymphocytes T spécifiques de l'antigène alimentaire ovalbumine peut promouvoir le développement d'une atrophie villositaire et l'activation des lymphocytes T CD8 ${ }^{+}$cytotoxiques chez des souris surexprimant I'IL-15 dans l'épithélium intestinal [13]. De plus, il convient de souligner le fait que, contrairement aux patients ayant une forme active de maladie cœliaque, un sous-groupe d'individus ayant une forme silencieuse de la maladie développent une réponse immunitaire adaptative contre le gluten en l'absence de destruction tissulaire, ne présentent pas d'entérocytes modifiés par le stress, et n'ont ni surexpression de l'IL-15 dans l'épithélium intestinal, ni lymphocytes intra-épithéliaux avec un phénotype cytotoxique $[14,15]$. Or, des observations effectuées chez les patients atteints de maladie cœliaque ont montré que les lymphocytes intraépithéliaux expriment des récepteurs NK (natural killer) qui sont activés par I'IL-15, et ont des capacités cytolytiques qui leur permettraient de lyser les cellules épithéliales exprimant leurs ligands, c'est-à-dire des molécules HLA de classe I non classiques induites par 
le stress et l'inflammation [16]. Ces observations ont guidé la mise au point d'un nouveau modèle murin physiopathologique de maladie cœliaque développant une atrophie villositaire après consommation de gluten.

Synergie entre la réponse immunitaire adaptative dirigée contre le gluten et la surexpression d'IL-15 dans l'épithélium intestinal Nous avons donc développé un modèle murin fondé sur l'hypothèse de la nécessité d'une réponse adaptative contre le gluten et de la présence simultanée d'un stress épithélial pour induire la destruction des cellules épithéliales intestinales par les lymphocytes intra-épithéliaux [17] (Figure 1). Nous avons tout d'abord analysé des souris qui surexpriment I'IL15 soit dans l'épithélium, soit dans le chorion de la muqueuse intestinale, ou dans ces deux compartiments à la fois. Des souris transgéniques $D Q 8-D^{d}-I L-$ $15 \mathrm{tg}$, qui surexpriment I'IL-15 dans le chorion et les ganglions mésentériques mais pas dans l'épithélium intestinal, développent, lorsqu'elles sont exposées au gluten par voie orale, une réponse inflammatoire de type $T_{H} l$ contre le gluten, et produisent des anticorps contre les peptides de gluten désamidés (DGP) sans toutefois présenter des lymphocytes intra-épithéliaux avec un profil cytotoxique augmenté $[17,18]$. $\varepsilon$ n revanche, des souris DQ8-villin-IL$15 \mathrm{tg}$, qui surexpriment I'IL-15 sous le contrôle du promoteur du gène codant la villine, une protéine spécifique de l'épithélium intestinal, ne développent pas d'immunité contre le gluten après en avoir consommé, alors qu'elles présentent une augmentation du nombre de lymphocytes intra-épithéliaux avec une forte expression de protéines effectrices cytotoxiques telles que le granzyme $B$ ou la perforine [17]. Ces souris ressemblent aux membres de la famille de patients atteints de maladie cœliaque qui présentent un stress épithélial avec surexpression d'IL-15 mais n'ont pas de lymphocytes intra-épithéliaux cytotoxiques
[15]. Aucun de ces deux modèles murins HLA-DQ8 transgéniques pour la production d'IL-15 ne développe d'atrophie villositaire intestinale. Afin de tester notre hypothèse de la nécessité d'une augmentation de la production d'IL-15 dans les deux compartiments de la muqueuse intestinale (épithélium et chorion) pour que surviennent des dommages tissulaires, nous avons produit des souris DQ8-D d -villin-IL-15tg.

\section{IL-15, gluten et HLA-D08}

conduisent à des lésions intestinales

Des souris DQ8-D ${ }^{d}$-villin-IL-15tg ayant consommé du gluten durant trente jours développent une atrophie villositaire de l'ileum, des anticorps IgG anti-DGP, et une réponse immunitaire contre le gluten de type $T_{H} l$ révélée par la présence d'anticorps de type IgG2c contre le gluten et par la production d'IFN- $\gamma$ dans le chorion de la muqueuse intestinale. Une augmentation du nombre de lymphocytes intra-épithéliaux est une caractéristique de la maladie cœliaque utilisée comme critère diagnostique de la maladie [2]. Chez les patients, les lymphocytes intra-épithéliaux des zones de lésions intestinales sécrètent de fortes quantités de protéines lytiques. De plus, ils expriment anormalement à leur surface des récepteurs NK activateurs tels que NKG2D et CD94/NKG2C, qui accroissent leur capacité à lyser les entérocytes présentant leurs ligands «de stress» (MICA/B et HLA- $\varepsilon$ ) [16]. Comme chez les patients, les souris DQ8$\mathrm{D}^{\mathrm{d}}$-villin-IL-15tg ayant consommé du gluten présentent une augmentation du nombre de lymphocytes intra-épithéliaux exprimant des récepteurs NK activateurs et des protéines cytotoxiques, et une augmentation de Rae-l et $\mathrm{Pa}-1$, qui sont les ligands des récepteurs NK chez la souris. Cela suggère que ces molécules de classe I non classiques sont induites quand la réponse immunitaire adaptative contre le gluten agit en synergie avec la surexpression d'IL-15 dans l'épithélium. De plus, ces résultats montrent que ce modèle murin, développe, après ingestion de gluten, toutes les manifestations de la maladie cœliaque: une immunité adaptative contre le gluten, une augmentation du nombre de lymphocytes intra-épithéliaux cytotoxiques, et une destruction du tissu intestinal. Comme chez l'homme, toutes ces caractéristiques disparaissent lorsque les animaux reprennent une alimentation sans gluten.

Le développement d'une atrophie villositaire dans ce modèle murin est fortement corrélé à l'expansion de lymphocytes intra-épithéliaux cytotoxiques, et n'a donc pas lieu lorsque les souris sont traitées avec un anticorps permettant d'éliminer les lymphocytes T CD8 ${ }^{+}$, ce qui confirme que les lymphocytes intra-épithéliaux cytotoxiques sont bien les cellules effectrices promouvant la destruction du tissu intestinal dans la maladie cœliaque. La maladie est en outre caractérisée par la présence, dans le chorion de la muqueuse intestinale, de lymphocytes $T$ spécifiques du gluten restreints par HLA-DQ2 ou HLA-DQ8, et qui expriment I'IFN- $\gamma$ et I'IL-21 [5, $19,20]$. Des souris $D^{d-}$ villin-IL-15tg, qui surexpriment I'IL-15 de la même manière que les souris modèles de la maladie cœliaque, mais n'expriment pas HLA-DQ8, ne développent pas d'atrophie villositaire après ingestion de gluten, ce qui indique qu'un haplotype du complexe majeur d'histocompatibilité prédisposant à la maladie cœliaque est également requis dans ce modèle animal. En accord avec le rôle central de l'enzyme TG2 et des lymphocytes T CD4 $4^{+}$dans la pathogénèse de la maladie cœliaque, les lésions intestinales n'apparaissent pas non plus chez les souris DQ8-D ${ }^{\mathrm{d}}$-villin-IL-15tg ayant consommé du gluten et traitées avec des inhibiteurs pharmacologiques de TG2 ou un anticorps dirigé contre les lymphocytes T CD4 ${ }^{+}$. De plus, l'analyse du profil cytotoxique des lymphocytes intra-épithéliaux chez les souris dépourvues de HLA-DQ8 ou de lymphocytes T CD4 ${ }^{+}$et exposées au gluten par voie orale confirme que HLA-DQ8 et lymphocytes $\mathrm{T} \mathrm{CD}^{+}$sont tous deux 


\section{RÉPONSE IMMUNITAIRE ADAPTATIVE}

Peptide

desamidé

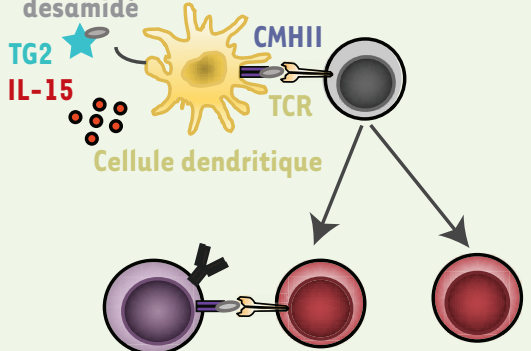

Lymphocyte B Lymphocyte $\mathrm{CD}^{+} \mathrm{T}_{\mathrm{H}} 1$

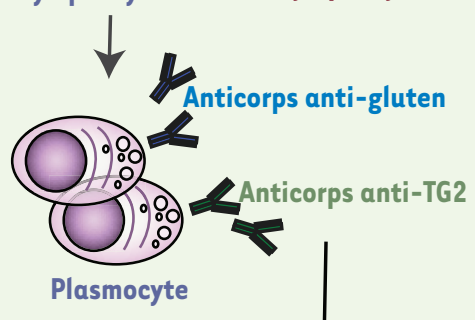

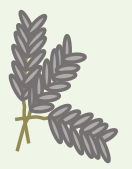

\section{ALTÉRATIONS AU NIVEAU DE L'ÉPITHÉLIUM}

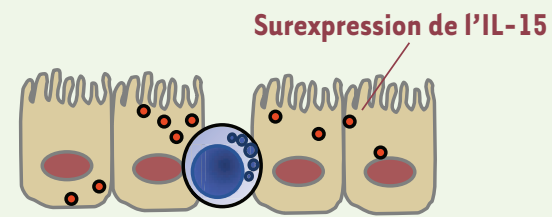

Lymphocyte T CD8* intra-épithélial

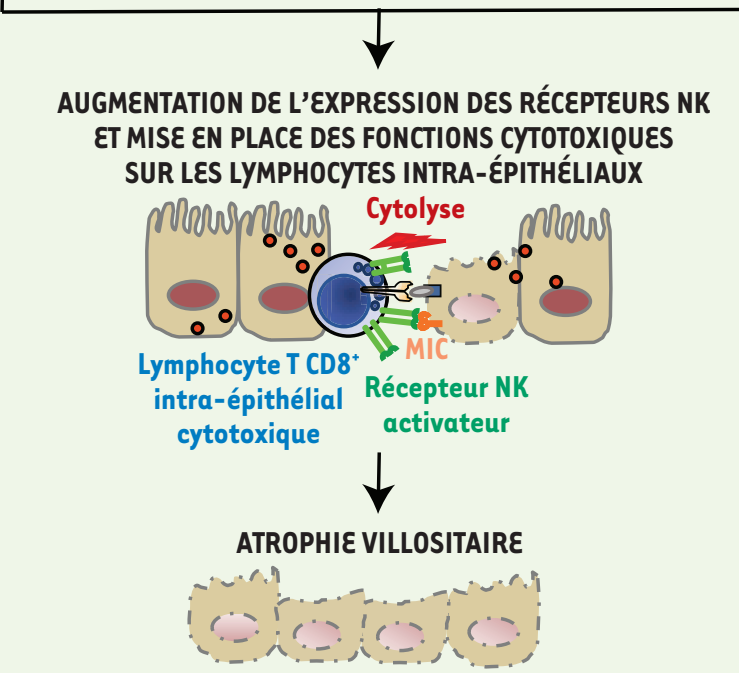

Figure 1. Une réponse immunitaire adaptative contre le gluten et la surexpression de l'IL-15 dans l'épithélium intestinal sont tous deux requis pour provoquer une maladie cœliaque avec atrophie villositaire. La maladie cœliaque se développe lorsque 1) une immunité adaptative spécifique du gluten s'établit en présence d'une surexpression de l'IL-15 dans le chorion de la muqueuse intestinale, c'est-à-dire que des lymphocytes T CD4 ${ }^{+}$spécifiques du gluten, restreints par les molécules HLA-DQ2 ou HLA-DQ8, et produisant des cytokines inflammatoires se différencient dans le chorion intestinal, et que des anticorps sont produits contre les peptides de gluten désamidés par la transglutaminase 2 (TG2) ainsi que des auto-anticorps contre TG2, et 2) les cellules épithéliales expriment des molécules « de stress » et surexpriment l'IL-15. Ces deux composants sont requis pour que les lymphocytes intraépithéliaux $\mathrm{CD} 8^{+}$acquièrent des propriétés effectrices cytotoxiques avec expression de protéines lytiques (granzyme B, perforine) et expression de récepteurs NK activateurs qui ont pour ligands les molécules du complexe majeur d'histocompatibilité de classe I non classiques, dont la synthèse est induite dans les entérocytes par le stress et l'inflammation. Ainsi, les lymphocytes intraépithéliaux CD8 $8^{+}$cytotoxiques lysent les entérocytes.

nécessaires pour conférer aux lymphocytes intra-épithéliaux des propriétés effectrices cytotoxiques et la capacité à éliminer les entérocytes.
Pour évaluer la contribution directe de l'IFN- $\gamma$ et de I'IL-2l produits par les lymphocytes $T$ spécifiques du gluten chez les patients atteints de maladie cœliaque, nous avons traité des souris DQ8-D ${ }^{d}$-villin-IL-15tg consommant du gluten avec un anticorps neutralisant I'IFN- $\gamma$ ou antagoniste du récepteur de 
I'IL-21. Alors que la production d'IFN- $\gamma$ est requise pour le développement d'une atrophie villositaire et l'expansion des lymphocytes $\mathrm{T} \mathrm{CD}^{+}$cytotoxiques, nos résultats montrent que I'IL-2l exerce surtout un rôle coopératif avec I'IFN- $\gamma$ dans la production d'anticorps dirigés contre le gluten.

Enfin, pour tenter de comprendre le processus faisant intervenir HLA-DQ8, IL-15, et le gluten, et aboutissant à l'atrophie villositaire, nous avons comparé le profil transcriptionnel de cellules isolées de l'épithélium et du chorion de la muqueuse intestinale de souris $D Q 8-D^{d}$ villin-IL-15tg, $D^{d}$-villin-IL-15tg, et DQ8tg ayant consommé du gluten. Nous avons trouvé un plus grand nombre de transcrits codant des protéines des voies de signalisation impliquées dans la présentation antigénique et l'activation des lymphocytes chez les souris DQ8-Dd-villin-IL15 tg que chez les souris $D^{d}$-villin-IL-15tg ou DQ8tg. Notre analyse révèle que l'interaction entre HLA-DQ8, I'IL-15 et le gluten est essentielle pour activer les voies de signalisation découlant de la réponse à I'IFN- $\gamma$. Enfin, la pertinence du modèle murin que nous avons développé pour de futures études précliniques applicables à I'homme est attestée par le fait que les changements dans l'expression des gènes chez les souris DQ8-Dd-villin-IL-15tg par rapport aux souris $D Q 8$ tg ne développant pas la maladie lorsqu'elles sont exposées au gluten sont fortement corrélés aux changements observés chez les patients ayant une forme active de la maladie cœliaque par rapport à des individus sains.

\section{Perspectives}

Cette étude présente le premier modèle animal de la maladie cœliaque dans lequel l'ingestion de gluten chez un hôte immunocompétent et génétiquement prédisposé est suffisante pour promouvoir le développement d'une atrophie villositaire dans l'intestin grêle comme chez les patients atteints de cette maladie [17]. Ce modèle murin reproduit la complexité de la maladie cœliaque, qui fait intervenir de manière coordonnée gluten, IL-15, HLA-DQ8, enzyme TG2, lymphocytes T $\mathrm{CD} 4^{+}$, et lymphocytes intra-épithéliaux $\mathrm{CD}^{+}$cytotoxiques, conduisant à une réponse inflammatoire dans le chorion de la muqueuse intestinale, à l'augmentation de la réponse à I'IFN- $\gamma$, et à l'augmentation du nombre de lymphocytes cytotoxiques. Ce modèle constitue donc un outil précieux, qui permettra non seulement d'accroître nos connaissances des mécanismes immunologiques impliqués dans la maladie cœliaque, mais aussi de tester de nouvelles stratégies thérapeutiques. $\diamond$ A new mouse model of coeliac disease with villous atrophy

\section{LIENS D'INTÉRÊT}

L'auteure déclare n'avoir aucun lien d'intérêt concernant les données publiées dans cet article.

\section{RÉFÉRENCES}

1. Abadie V, Sollid LM, Barreiro LB, Jabri B. Integration of genetic and immunological insights into a mode of celiac disease pathogenesis. Annu Rev Immunol $2011 ; 29$ : 493-525.

2. Husby S, Koletzko S, Korponay-Szabo I, et al. European society paediatric gastroenterology, hepatology and nutrition guidelines for diagnosing coeliac disease 2020. J Pediatr Gastroenterol Nutr $2020 ; 70: 141-56$.

3. Murray JA, Watson T, Clearman B, Mitros F. Effect of a gluten-free diet on gastrointestinal symptoms in celiac disease. Am J Clin Nutr 2004 ; 79 : 669-73.

4. Daum S, Cellier C, Mulder C). Refractory coeliac disease. Best Pract Res Clin Gastroenterol 2005 ; 19 : 413-24.
5. Nilsen EM, Jahnsen FL, Lundin KE, et al. Gluten induces an intestinal cytokine response strongly dominated by interferon gamma in patients with celiac disease. Gastroenterology 1998 ; 115 : 551-63.

6. Dieterich W, Laag $\varepsilon$, Schopper H, et al. Autoantibodies to tissue transglutaminase as predictors of celiac disease. Gastroenterology 1998 ; 115 : 1317-21.

7. Molberg 0 , McAdam SN, Korner $\mathrm{R}$, et al. Tissue transglutaminase selectively modifies gliadin peptides that are recognized by gut-derived T cells in celiac disease. Nat Med 1998; $4: 713-7$.

8. Van de Wal Y, Kooy Y, van Veelen P, et al. Selective deamidation by tissue transglutaminase strongly enhances gliadin-specific T cell reactivity. J Immunol $1998 ; 161: 1585-8$.

9. Black KE, Murray JA, David CS: HLA-DQ determines the response to exogenous wheat proteins: a model of gluten sensitivity in transgenic knockout mice. J Immunol 2002 ; 169 : 5595-600.

10. de Kauwe AL, Chen Z, Anderson RP, et al. Resistance to celiac disease in humanized HLA-DR3-DQ2transgenic mice expressing specific anti-gliadin CD4 ${ }^{+}$ T cells. J Immunol 2009 ; 182 : 7440-50.

11. Du Pre MF, Kozijn AE, van Berkel LA, et al. Tolerance to ingested deamidated gliadin in mice is maintained by splenic, type 1 regulatory T cells. Gastroenterology $2011 ; 141: 610-20$.

12. Verdu EF, Huang X, Natividad J, et al. Gliadindependent neuromuscular and epithelial secretory responses in gluten-sensitive HLA-DQ8 transgenic mice. Am J Physiol Gastrointest Liver Physiol 2008 ; 294 : G217-25.

13. Korneychuk N, Ramiro-Puig E, Ettersperger J, et al. Interleukin 15 and $\mathrm{CD}^{+}{ }^{+} \mathrm{T}$ cells cooperate to promote small intestinal enteropathy in response to dietary antigen. Gastroenterology 2014 ; 146 : 1017-27.

14. Mention JJ, Ben Ahmed M, Begue B, et al. Interleukin 15: a key to disrupted intraepithelial lymphocyte homeostasis and lymphomagenesis in celiac disease. Gastroenterology 2003 ; $125: 730-45$.

15. Setty M, Discepolo V, Abadie V, et al. Distinct and synergistic contributions of epithelial stress and adaptive immunity to functions of intraepithelial killer cells and active celiac disease. Gastroenterology $2015 ; 149$ : 681-91.

16. Abadie V, Discepolo V, Jabri B. Intraepithelial lymphocytes in celiac disease immunopathology. Semin Immunopathol 2012 ; 34 : 551-66.

17. Abadie V, Kim SM, Lejeune T, et al. IL-15, gluten and HLA-DQ8 drive tissue destruction in coeliac disease. Nature $2020 ; 578: 600-4$.

18. DePaolo RW, Abadie V, Tang F, et al. Co-adjuvant effects of retinoic acid and IL-15 induce inflammatory immunity to dietary antigens. Nature $2011 ; 471: 220-4$.

19. Bodd M, Raki M, Tollefsen S, et al. HLA-DQ2-restricted gluten-reactive $T$ cells produce IL- 21 but not IL- 17 or IL-22. Mucosal Immunol 2012 ; 3 : 594-601.

20. Fina D, Sarra M, Caruso R, et al. Interleukin 21 contributes to the mucosal Thelper cell type 1 response in coeliac disease. Gut 2008 ; 57 : 887-92.

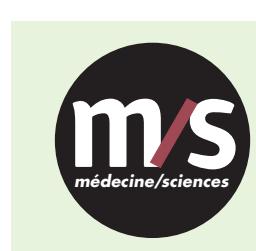

Tarifs d'abonnement m/s - 2020

Abonnez-vous

à médecine/sciences
$>$ Grâce à $m / s$, vivez en direct les progrès des sciences biologiques et médicales

Bulletin d'abonnement page 1098 dans ce numéro de $\mathrm{m} / \mathrm{s}$

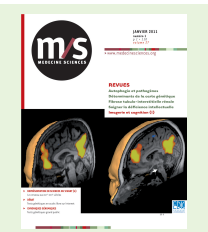

